\title{
ANÁLISE ECONÔMICA DE SISTEMAS AGROFLORESTAIS NA AMAZÔNIA OCIDENTAL, MACHADINHO D'OESTE- RO' ${ }^{1}$
}

Michelliny de Matos Bentes-Gama², Márcio Lopes da Silva ${ }^{3}$, Luciano Javier Montoya Vilcahuamán ${ }^{4}$ e Marilia Locatelli ${ }^{5}$

\begin{abstract}
RESUMO - O objetivo deste estudo foi realizar a análise financeira e a simulação de risco de investimento em sistemas agroflorestais (SAFs) implantados em 1987, no Campo Experimental da Embrapa Rondônia, localizado no município de Machadinho d'Oeste, RO. A análise financeira foi realizada mediante os métodos de avaliação de projetos florestais, e para a análise de risco utilizou-se a técnica de simulação de Monte Carlo, mediante o programa @ RISK. Entre os arranjos testados, o SAF $\mathrm{T}_{1 \text { Castanha-do-brasil-banana-pimenta-do-reino-cupuacu }}$ apresentou o melhor

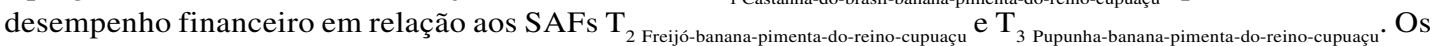
custos com tratos culturais e colheita representaram mais de $70 \%$ da composição dos custos totais, e a participação da mão-de-obra foi superior a 50\% nas fases de preparo da área e de manutenção (tratos culturais) dos SAFs. A simulação da análise de risco indicou que as variáveis que afetaram o Valor Presente Líquido no Horizonte Infinito (VPL*), de acordo com a ordem de importância (R), foram: taxa de desconto, preço do fruto de cupuaçu (Theobroma grandiflorum), custo de colheita, preço da madeira de castanha-do-brasil (Bertholletia excelsa) e o custo de tratos

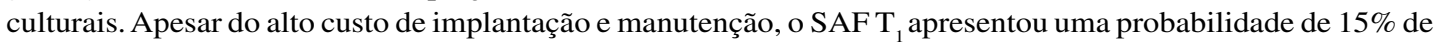
os valores do Valor Presente Líquido (VPL) se concentrarem em torno de R $\$ 35.000$ ha $^{-1}$.ano ${ }^{-1}$.
\end{abstract}

Palavras-chave: Análise financeira, fluxo de caixa e rendimento agroflorestal.

\section{ECONOMIC ANALYSIS OF AGROFORESTRY SYSTEMS IN EASTERN AMAZONIA, MACHADINHO D'OESTE- RO, BRAZIL}

\begin{abstract}
The objective of this study was to carry out the financial analysis and the risk of investment simulation in agroforestry systems (AFSs) established in 1987 in the Experimental Field of Embrapa Rondônia, located in the County of Machadinho d'Oeste, RO. Financial analysis was made through the evaluation of results from the forestry enterprise evaluation methods, and the risk analysis was carried out through the Monte Carlo simulation technique by @ RISK software. Among the arrangements tested, $T_{1}$ pepper-Сupuacu was the agroforestry system $(A F S)$ with the best financial results, comparing to $T_{2 \text { Frreijo wood-banana- }}$ black pepper-cupuacu and $T_{3 \text { Pupunha palm-banana-black pepper-cupuacu }}$. The management and harvesting costs represented more than $70 \%$ of the total cost composition; and the labor cost participation was higher than $50 \%$ in the site preparation and long-term maintenance phases of the agroforestry systems. The risk analysis simulation showed that the variables which affected Infinite Horizon Net Present Value (NPV*), according to the ranking of importance $(R)$, were: discount rate, price of cupuaçu fruits (Theobroma grandiflorum), harvesting cost, price of Brazil
\end{abstract}

\footnotetext{
${ }^{1}$ Recebido em $1^{\circ} .06 .2004$ e aceito para publicação em 20.04.2005.

2 Embrapa Rondônia, Setor Técnico-Científico, BR 364, km 5,5, Cx.Postal 406, 78900-970. Porto Velho-RO. E-mail: <mbgama@cpafro.embrapa.br>.

${ }^{3}$ Departamento de Engenharia Florestal da Universidade Federal de Viçosa, 36570-000. Viçosa-MG.

${ }^{4}$ Embrapa Florestas, Estrada da Ribeira, km 111, Cx.Postal 319, 83.411-000. Colombo, PR.

${ }^{5}$ Embrapa Rondônia.
} 
nut (Bertholletia excelsa) wood, and long-term maintenance costs. Even though the high establishment and the long-term maintenance costs, AFS $T_{1 \text { Castanha-do-brasil-Banana-black pepper-cupuacu }}$ showed a $15 \%$ probability that Net Present Value (NPV) value could be concentrated around $R \$ 35.000 \mathrm{ha}^{-1}$ year $\mathrm{r}^{-1}$.

Key words: Financial analysis, cash flow and agroforestry incomes.

\section{INTRODUÇÃO}

Ademanda pela pesquisa agroflorestal na Amazônia, e em Rondônia, é crescente devido à necessidade de alternativas tecnológicas e inovações que visem ao desenvolvimento socioeconômico regional e substituam a agricultura migratória, dois dos grandes desafios que ainda persistem na região. Dessa maneira, estudos específicos sobre a viabilidade econômica de investimento em sistemas agroflorestais (SAFs), como alternativa para a diversificação da produção e renda, e recuperação ambiental em regiões onde se pratica a agricultura tropical têm sido cada vez mais necessários.

A diversificação de cultivos mediante os SAFs é uma fonte estratégica de produção de alimentos diante do monocultivo, embora estes também estejam suscetíveis às variações do desempenho das culturas selecionadas, bem como às flutuações dos preços de mercado (OLIVEIRA e VOSTI, 1997; RAMÍREZ et al., 2001), entre outros fatores tecnológicos e econômicos.

Apesar da concordância de que os SAFs apresentam vantagens ecológicas e podem reduzir o risco de investimento em uma só cultura, constatase que estes representam uma atividade complexa que apresenta tantos riscos e incertezas como outras atividades agrícolas e florestais mais conhecidas; partindo daí a importância de se fazerem avaliações econômicas sob condições de risco para subsidiar os agentes de financiamento, técnicos e produtores nesse tipo de investimento na Amazônia.

Algumas das experiências com análise financeira de SAFs amazônicos (OLIVEIRAe VOSTI, 1997; SILVA, 2000; SÁ et al., 2000; SANTOS, 2000; ARCO-VERDE et al., 2003) confirmam que as associações de cultivos arbóreos, perenes e anuais proporcionam uma rápida recuperação do capital investido, com geração de renda imediata nos primeiros anos pela comercialização de culturas agrícolas de ciclos curto e médio, e ao longo da duração do sistema com a venda de diversos produtos, havendo destaque para a produção de frutas e madeira.
Embora sejam resultados válidos, ainda há um enfoque reduzido de estudos quanto à avaliação econômica dos SAFs na Amazônia. Portanto, a especificidade dos arranjos e culturas utilizados nos SAFs regionais faz com que seja necessário o acompanhamento da produção e do desempenho financeiro, bem como da aplicação de novas tecnologias, além de estudos das tendências de mercado, a fím de gerar informações confiáveis aos produtores e técnicos locais e, assim, promover uma adoção mais efetiva desse tipo de sistema de produção.

O objetivo deste estudo foi realizar a análise financeira e a simulação de risco de investimento em sistemas agroflorestais implantados em 1987, no Campo Experimental da Embrapa no município de Machadinho d'Oeste, Rondônia.

\section{MATERIAL E MÉTODOS}

\section{1. Área de estudo}

Foram utilizados dados provenientes do experimento Teste de sistemas agroflorestais para a região de Machadinho (RO), instalado em fevereiro de 1987 no Campo Experimental da Embrapa, localizado no município de Machadinho d'Oeste, Rondônia, que apresenta as seguintes características gerais, conforme Miranda et al. (2002): está localizado entre os muncípios de Ariquemes e Jaru, a aproximadamente $400 \mathrm{~km}$ da capital Porto Velho ( $61^{\circ} 47^{\prime} \mathrm{S}$ e $\left.63^{\circ} 00^{\prime} \mathrm{W}\right)$; apresenta clima do tipo Am, de acordo com a classifcação de Köppen, com chuvas do tipo monção; com estação chuvosa de dezembro a março, com precipitações anuais em torno de $2.000 \mathrm{~mm}$; sendo a estação seca de junho a agosto; com temperatura média anual de $24^{\circ} \mathrm{C}$ e umidade relativa do ar entre 80 e $85 \%$.

O experimento abrangeu uma área de 4,68 ha, que apresenta relevo plano e solo classificado como Latossolo Amarelo textura argilosa (LOCATELLI, 1987). O delineamento experimental foi o de blocos casualizados (oito tratamentos e quatro blocos), sendo três sistemas 
agroflorestais (SAFs) e cinco monocultivos. Os SAFs foram compostos pelas espécies: castanha-do-brasil (Bertholletia excelsa H.B.K.), freijó (Cordia alliodora (Ruiz \& Pav.) Oken), pupunha (Bactris gasipaes Kunth), cupuaçu (Theobroma grandiflorum (Willd. ex Spreng.) K. Schum.), banana (Musa spp.) e pimenta-do-reino (Piper nigrum L.).

A análise seguiu o esquema de parcelas subdivididas, estudando-se nas parcelas os sistemas de produção e, nas subparcelas, o tempo. Neste trabalho foram analisados apenas os sistemas agroflorestais (Quadro 1).

\subsection{Variáveis}

As variáveis de produção por espécie utilizadas neste estudo foram: Castanha-do-brasil: volume total de madeira, em $\mathrm{m}^{3}$, e número de frutos por planta; Freijó: volume total de madeira, em $\mathrm{m}^{3}$; Pupunha: peso do cacho, em kg; Сupuaçu: peso do fruto, em kg; Banana: peso do cacho, em kg; e Pimenta-do-reino: peso de grãos secos, em kg. Para a estimativa da receita com madeira das espécies perenes castanha-do-brasil e freijó, consideraram-se o volume comercial, o preço médio de mercado da venda da madeira em pé na região (OLIVEIRA et al., 1999) e a colheita a partir do ano 15. Nas demais espécies, levou-se em conta o preço médio comercializado na região, mediante dados obtidos no IBGE (2001) e na revisão de literatura (Quadro 2).
Quadro 2 - Preços dos produtos considerados nas análises dos sistemas agroflorestais $\left(\mathrm{T}_{1}, \mathrm{~T}_{2} \mathrm{e} \mathrm{T}_{3}\right)$ testados em Machadinho d'Oeste, $\mathrm{RO}-1987$ a 2002

Table 2 - Price of the products considered in the anlysis of the agroforestry systems $\left(T_{1}, T_{2}\right.$ and $\left.T_{3}\right)$ tested in Machadinho d'Oeste, RO- 1987 to 2002

\begin{tabular}{lccc}
\hline Espécie & Produto & Unidade & Preço $(\mathrm{R} \$)$ \\
\hline Castanha-do-brasil & Fruto & und. & 0,50 \\
& Madeira & $\mathrm{m}^{3}$ & 100,00 \\
Freijó & Madeira & $\mathrm{m}^{3}$ & 100,00 \\
Pupunha & Cacho & $\mathrm{kg}$ & 0,25 \\
Banana & Cacho & $\mathrm{kg}$ & 0,30 \\
Pimenta-do-reino & Grãos secos & $\mathrm{kg}$ & 1,20 \\
Cupuaçu & Fruto & $\mathrm{kg}$ & 1,00 \\
\hline
\end{tabular}

\subsection{Composição dos custos}

Os custos para a avaliação econômica deste estudo envolveram os coeficientes de mão-de-obra, insumos e equipamentos necessários à realização de cada atividade, e foram determinados a partir do desenvolvimento de fórmulas do valor atual para os horizontes de planejamento estabelecidos. As informações sobre os coeficientes técnicos e as atividades realizadas em cada SAF foram obtidas do acompanhamento das atividades realizadas no Campo Experimental da Embrapa Rondônia, em Machadinho d'Oeste (Quadro 3). Informações complementares foram obtidas na revisão de literatura, entre os quais: Calzavara (1989); Venturieri (1993); Carvalho et al. (1999); Bergo e Lunz (2000); Sá et al. (2001); Monteiro (2002); Yamada e Gholz (2002) e Duarte e Poltronieri (2003).

Quadro 1 - Informações sobre os sistemas agroflorestais $\left(\mathrm{T}_{1}, \mathrm{~T}_{2}\right.$ e $\left.\mathrm{T}_{3}\right)$ testados em Machadinho d'Oeste, RO - 1987 a 2002

Table 1 - Information about the agroforestry systems $\left(T_{1}, T_{2}\right.$ and $\left.T_{3}\right)$ tested in Machadinho d'Oeste, RO - 1987 to 2002

\begin{tabular}{|c|c|c|c|c|c|c|}
\hline Sistema $^{(1)}$ & $\begin{array}{c}\text { Espécie }{ }^{(2)} \\
\text { Parcela }\left(\mathrm{m}^{2}\right)\end{array}$ & $\begin{array}{c}\text { Área da } \\
(\mathrm{m})\end{array}$ & $\begin{array}{c}\text { Espaçamento } \\
\text { por Planta }\left(\mathrm{m}^{2}\right)\end{array}$ & $\begin{array}{c}\text { Área Ocupada } \\
\text { por Parcela }\end{array}$ & $\begin{array}{c}\text { № de Plantas } \\
\text { ha }^{-1}\end{array}$ & № de Plantas \\
\hline \multirow{4}{*}{$\mathrm{T}_{1}$} & $\mathrm{Ca}$ & \multirow{4}{*}{3.600} & $12 \times 12$ & 144 & 25 & 69 \\
\hline & $\mathrm{Ba}$ & & $6 \times 6$ & 36 & 72 & 278 \\
\hline & $\mathrm{Pm}$ & & $6 \times 2$ & 12 & 78 & 833 \\
\hline & $\mathrm{Cp}$ & & $6 \times 6$ & $18^{(3)}$ & 56 & 556 \\
\hline \multirow{4}{*}{$\mathrm{T}_{2}$} & $\mathrm{Fr}$ & \multirow{4}{*}{900} & $6 \times 6$ & 36 & 25 & 278 \\
\hline & $\mathrm{Ba}$ & & $6 \times 6$ & 36 & 20 & 278 \\
\hline & $\mathrm{Pm}$ & & $6 \times 6$ & 36 & 78 & 278 \\
\hline & $\mathrm{Cp}$ & & $6 \times 6$ & 18 & 16 & 556 \\
\hline \multirow{4}{*}{$\mathrm{T}_{3}$} & $\mathrm{Pu}$ & \multirow{4}{*}{900} & $6 \times 6$ & 36 & 25 & 278 \\
\hline & $\mathrm{Ba}$ & & $6 \times 6$ & 36 & 20 & 278 \\
\hline & $\mathrm{Pm}$ & & $6 \times 6$ & 36 & 78 & 278 \\
\hline & $\mathrm{Cp}$ & & $6 \times 6$ & 18 & 16 & 558 \\
\hline
\end{tabular}

${ }^{(1)} \mathrm{T}_{1}$ : castanha-do-brasil, banana, pimenta-do-reino e cupuaçu, $\mathrm{T}_{2}$ freijó, banana, pimenta-do-reino e cupuaçu, $\mathrm{T}_{3}$ : pupunha, banana, pimentado-reino e cupuaçu; (2) Ca: castanha-do-brasil, Ba: banana, Pm: pimenta-do-reino, Cp: cupuaçu, Fr - freijó e Pu: pupunha. ${ }^{(3)} \mathrm{Refere-se}$ ao arranjo em quincôncio dessa espécie nos tratamentos $\mathrm{T}_{1}, \mathrm{~T}_{2}$ e $\mathrm{T}_{3}$. 
Quadro 3 - Resumo das atividades de estabelecimento e manutenção dos sistemas agroflorestais $\left(T_{1}, T_{2}\right.$ e $\left.T_{3}\right)$ testados em Machadinho d'Oeste, RO - 1987 a 2002

Table 3 - Summary of the activities of establishment and maintenance of the agroforestry systems $\left(T_{1}, T_{2}\right.$ and $\left.T_{3}\right)$ tested

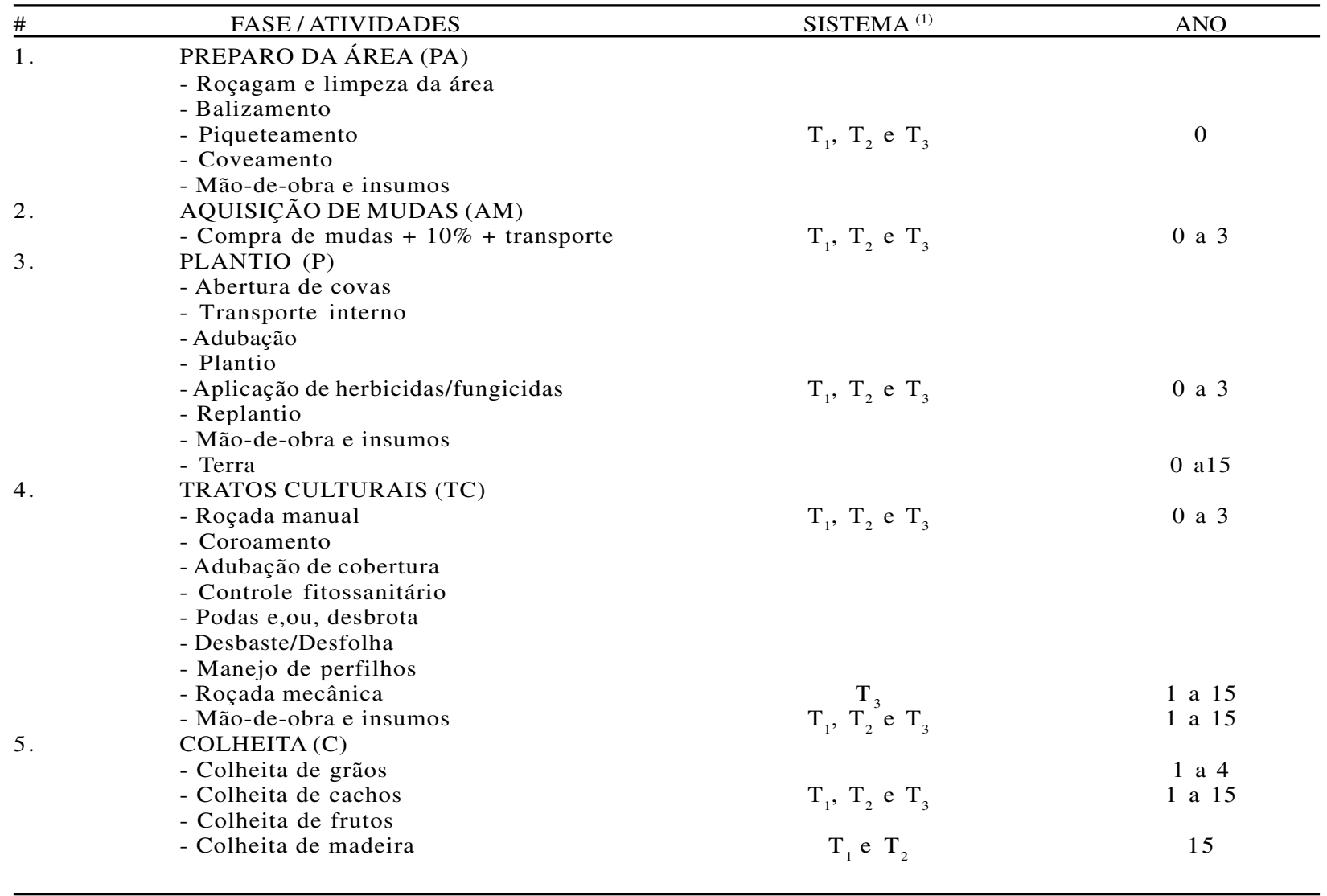

(1) $\mathrm{T}_{1}$ : castanha-do-brasil, banana, pimenta-do-reino e cupuaçu; $\mathrm{T}_{2}$ : freijó, banana, pimenta-do-reino e cupuaçu; e $\mathrm{T}_{3}$ : pupunha, banana, pimenta-do-reino e cupuaçu.

\subsection{Fluxos de caixa}

Em cada sistema foram isolados os fluxos de receitas e custos das culturas, que foram avaliados a partir da aplicação simultânea de métodos de avaliação financeira, utilizando-se como referência um único momento no horizonte de tempo, conforme Santos (2000), para o qual todos os valores foram atualizados mediante fórmulas financeiras de acumulação ou desconto de juros.

\subsection{Análise financeira}

A análise financeira foi realizada com a finalidade de verificar se a renda gerada pelos SAFs remunera ou não o capital investido, mediante os métodos de avaliação de projetos vistos nos tópicos subseqüentes.

\subsubsection{Valor Presente Líquido - VPL}

A viabilidade econômica de um projeto analisado pelo VPLé indicada pela diferença positiva entre receitas e custos atualizados para determinada taxa de dêsconto (REZENDE e OLIVEIRA, 2001; SILVA et al., 2002).

$$
V P L=\sum_{j=1}^{n} \frac{R_{j}}{(1+i)^{j}}-\sum_{j=1}^{n} \frac{C_{j}}{(1+i)^{j}}
$$

em que $\underline{R}_{j}=$ receitas no período $j ; C_{j}=$ custos no período $j ; i=$ taxa de desconto; $j=$ período de ocorrência de $R_{j}$ e $C_{j}$; e $n=$ duração do projeto, em anos, ou em número de períodos de tempo.

\subsubsection{Valor Presente Líquido para o Horizonte Infinito - VPL*}

Considerou-se também o VPL de uma série de 
infinitos ciclos da cultura (VPL*). Dessa forma, puderamse comparar, conforme Santos et al. (2002), os projetos ou tratamentos com ciclos de durações diferentes.

$$
V P L^{*}=\frac{V P L(1+i)^{p}}{\left[(1+i)^{p}-1\right]}=\frac{V L F}{\left[(1+i)^{p}-1\right]}
$$

em que $V P L^{*}=$ valor presente líquido da série infinita de cultivos; VPL = valor presente líquido de um ciclo de cultivo que se repete perpetuamente; $V L F=$ valor futuro líquido, no final de um ciclo de cultivo, que se repete perpetuamente; $p=$ período ou ciclo da cultura (rotação); e $i=$ taxa de desconto.

\subsubsection{Valor Anual Equivalente - VAE}

O Valor Anual Equivalente (VAE) é a parcela periódica e constante necessária ao pagamento de uma quantia igual ao VPL da opção de investimento em análise ao longo de sua vida útil. O projeto será considerado economicamente viável quanto maior for o valor do benefício periódico equivalente (REZENDE e OLIVEIRA, 2001; SILVA et al., 2002).

$$
V A E=\frac{V P L \cdot i}{1-(1+i)^{-n}}
$$

em que $V P L=$ valor presente líquido; $n=$ duração do ciclo ou rotação, em anos.

\subsubsection{Razão Benefício/Custo - B/C}

Este método consiste em determinar a relação entre o valor presente dos benefícios e o valor presente dos custos, para determinada taxa de juros ou descontos. Um projeto é considerado viável economicamente se $B / C>1$. Entre dois ou mais projetos, o mais viável é aquele que apresentar o maior valor de $B / C$ (REZENDE e OLIVEIRA, 2001). Quando $B / C=1$, resulta em $V P L$ $B / C=0$; nesse caso, a $T I R$ associada a um projeto pode também ser determinada como sendo a taxa que faz com que $B / C=1$.

$$
B / C=\frac{\sum_{j=0}^{n} R_{j}(1+i)^{-j}}{\sum_{j=0}^{n} C_{j}(1+i)^{-j}}
$$

em que: $R_{j}=$ receita no final do ano $j ; C_{i}=$ custo no final do ano $j$; e $n=$ duração do projeto, em anos.

\subsubsection{Taxa Interna de Retorno - TIR}

A TIR é a taxa de desconto que iguala o valor atual das receitas futuras ao valor atual dos custos futuros do projeto, constituindo uma medida relativa que reflete o aumento no valor do investimento ao longo do tempo, com base nos recursos requeridos para produzir o fluxo de receitas (REZENDE e OLIVEIRA, 2001; SILVA et al., 2002).

$$
B / C=\frac{\sum_{j=0}^{n} R_{j}(1+i)^{-j}}{\sum_{j=0}^{n} C_{j}(1+i)^{-j}}
$$

em que $T I R=$ Taxa interna de retorno; as demais variáveis já foram definidas.

Nesta análise financeira, considerou-se a aplicação de taxas reais de desconto de $10 \%$ ao ano $(i=10 \%$ a.a.), conforme Oliveira e Vosti (1997) e Sá et al. (2000). No cálculo do custo anual da terra foi considerada a mesma taxa de desconto. Os custos das atividades e os preços dos produtos foram levantados em outubro de 2003, época em que o dólar estava cotado em 1 US $\$=$ R $\$$ 2,90 . Os dados foram analisados tendo como auxílio o software Excel 98.

\subsection{Análise de risco}

De maneira geral, os benefícios e custos associados ao fluxo de caixa de projetos de investimento normalmente são considerados conhecidos, caracterizando o que se conhece como procedimento de análise determinística; que, apesar de sua praticidade, leva a uma simplificação e, ou, a superestimativa de informações que nem sempre são conhecidas com certeza no momento da análise, como preços, quantidades, e rendimentos, entre outros. Uma forma de minimizar esse problema é adotar uma análise em condições de risco, em que se utilizam distribuições de probabilidade associadas aos indicadores de desempenho do projeto.

$\mathrm{Na}$ análise de risco de investimento neste estudo, optou-se por utilizar as informações do SAF de melhor desempenho financeiro. Os dados foram analisados mediante o software @RISK (PALISADE CORPORATION, 1992). Esse programa permite a aplicação do método de Monte Carlo para simular valores para as variáveis aleatórias receita e custo e, em decorrência dos valores aleatórios gerados, obter valores para a variável lucro. Foram definidas 10.000 iterações e consideradas como variáveis de entrada (inputs): preço da madeira do componente florestal, preço do principal componente agrícola, taxa de desconto e os dois principais componentes de custos. Consideraram-se

R. Árvore, Viçosa-MG, v.29, n.3, p.401-411, 2005 
ainda variações entre $-20 \%$ e $+20 \%$ nessas variáveis, com base na distribuição de probabilidade triangular, conforme Rodriguez (1987). Os indicadores financeiros VPL, TIR, VPL*, VAE e B/C foram tomados como variáveis de saída (outputs).

\section{RESULTADOS E DISCUSSÃO}

\subsection{Fluxos de caixa}

Os sistemas agroflorestais (SAFs) analisados apresentaram fluxos de caixa com regularidade de receitas ao longo do período considerado (Figura 1). OSAFT foi o que apresentou resultados positivos desde o ano 1 , gerando uma renda líquida total de $\mathrm{R} \$ 13.045,62 \mathrm{ha}^{-1}$ no ano 15 e outra média mensal de $\mathrm{R} \$ 1.087,08$. Uma renda líquida total inferior foi observada no $\mathrm{SAF}_{2}(\mathrm{R} \$ 5.609,89)$, no período avaliado, que só proporcionou retornos postivos a partir do ano 5, demonstrando que a combinação das espécies desse sistema não apresentou rendimentos tão satisfatórios quando comparados com os do SAF $\mathrm{T}_{1}$; embora tenham sido razoáveis em comparação com os resultados obtidos por Oliveira e Vosti (1997) para SAFs implantados em Ouro Preto d'Oeste, RO, formados por freijó (6 m x $6 \mathrm{~m})$, cupuaçu ( $6 \mathrm{~m} \times 6 \mathrm{~m})$ e pimenta-do-reino ( $6 \mathrm{~m} \mathrm{x}$ $3 \mathrm{~m})$, que geraram receita de $\mathrm{R} \$ 6.540,00 . \mathrm{ha}^{-1}$ no período de 20 anos.

A forte competição observada entre as espécies cupuaçu e pupunha no SAF $\mathrm{T}_{3}$ (LOCATELLI, 2001;

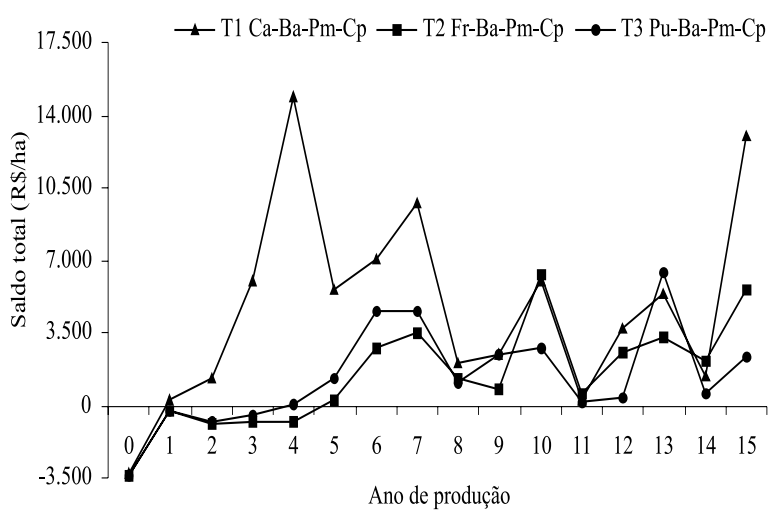

Figura 1 - Fluxo de caixa (saldo total) dos sistemas agroflorestais $\left(\mathrm{T}_{1}, \mathrm{~T}_{2} \mathrm{e}_{3}\right)$ testados em Machadinho d'Oeste, RO - 1987 a 2002.

Figure 1 - Cash flow (total sum) of the agroforestry systems $\left(T_{1}, T_{2}\right.$ and $\left.T_{3}\right)$ tested in Machadinho d'Oeste, $R O$ -1987 to 2002 .
GAMA, 2003) ocasionou a oscilação da renda ao longo do período avaliado, que atingiu um valor líquido total de R $\$ 2.339,99$ no ano 15 , o menor de todos os SAFs nesse ano, embora no ano 13 tivesse gerado renda líquida total de $\mathrm{R} \$ 6.385,89$, inferior a $15 \%$ daquela obtida no $\mathrm{SAF} \mathrm{T}_{1}$ e superior em aproximadamente $52 \%$ àquela gerada pelo $\mathrm{SAF}_{2}$ no mesmo ano.

A renda líquida total do último ano do $\mathrm{SAF} \mathrm{T}_{3}$ foi $34 \%$ inferior àquela encontrada por Sá et al. (2000) em sistemas agroflorestais implantados em Nova Califórnia, RO, com a combinação cupuaçu (120 plantas/ ha), pupunha (198 plantas/ha) e castanha-do-brasil (40 plantas/ha). Tais resultados indicaram que, mesmo com alguns resultados econômicos e ecológicos pouco favoráveis, e com elevados custos de implantação e manutenção, o plantio de SAFs que incluem a pupunha como espécie perene ainda se apresenta como alternativa econômica viável para a produção de frutos, tendo em vista a ocorrência de saldos positivos em 12 anos de duração do projeto com o arranjo testado, devendose, portanto, aplicar inovações tecnológicas que considerem as questões de espaçamento e manejo, entre outras exigências de cada espécie componente, a fim de otimizar o rendimento econômico.

\subsection{Custos dos sistemas}

Observou-se a mesma tendência da participação dos componentes do custo total (CT) - preparo da área, compra de mudas, plantio, tratos culturais e colheita em todos os SAFs (Figura 2). Durante o período de avaliação considerado, os custos totais nesses sistemas corresponderam a $\mathrm{R} \$ 18.254,90\left(\mathrm{SAF} \mathrm{T}_{1}\right), \mathrm{R} \$ 19.008,50$ $\left(\mathrm{SAF} \mathrm{T}_{2}\right)$ e R $\$ 20.333,80\left(\mathrm{SAF} \mathrm{T}_{3}\right)$. O custo com tratos culturais em todos os SAFs apresentou-se como o mais elevado, correspondendo a mais de $40 \%$ dos custos totais nessa fase, seguido pelos custos de colheita, superiores a $30 \%$ dos custos totais. Esse resultado é justificado pelo aumento de uso de máquinas, equipamentos e mão-de-obra nas referidas fases.

A participação da mão-de-obra nos três SAFs foi maior no preparo da área, correspondendo a mais de $50 \%$ dos custos totais. A segunda maior participação da mão-de-obra em todos os SAFs referiu-se à manutenção (tratos culturais) dos sistemas. Nos SAFs $\mathrm{T}_{1}$ e $\mathrm{T}_{2}$, a terceira maior participação da mão-de-obra ocorreu na fase de plantio, enquanto no $\mathrm{SAF} \mathrm{T}_{3}$ essa participação concentrou-se na fase de colheita (Figura 3). 

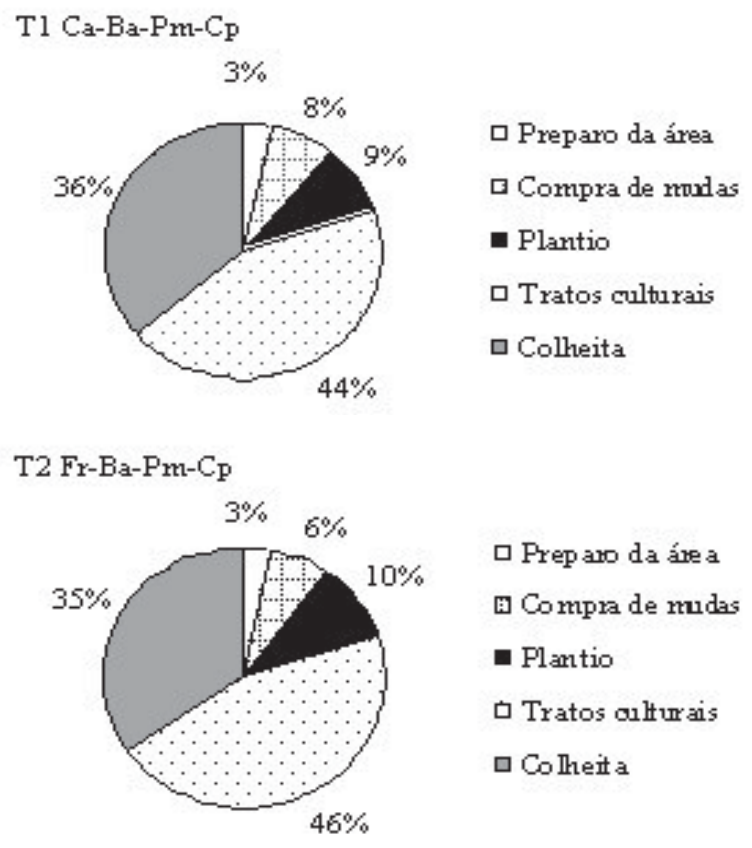

T3 Pu-Ba-Pm-Cp

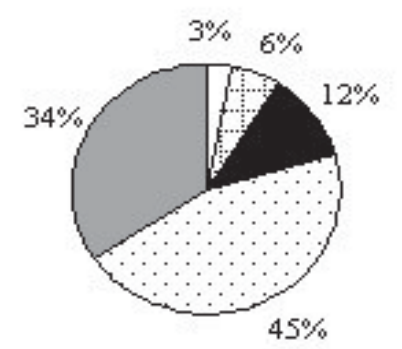

口 Prep aro da área

v. Compra de mudas

- Plantio

口 Tratos oulturais

口 Colheita

$\mathrm{R} \$ 45.865,26 \mathrm{ha}^{-1} \mathrm{ano}^{-1}$, com um VAE de R $\$ 4.586,53 \mathrm{ha}^{-1}$ $\mathrm{ano}^{-1}$, correspondente a um lucro de cinco a seis vezes maior aos obtidos com as receitas descontadas nos SAFs $\mathrm{T}_{3}$ e $\mathrm{T}_{2}$, respectivamente. A diversificação das receitas geradas pelo $\mathrm{SAF} \mathrm{T}_{1}$, com saldo positivo já a partir do ano 1 até o último ano, gerou um valor elevado da TIR, indicando a boa rentabilidade anual do capital investido nesse projeto (Quadro 4). Tais resultados são compatíveis aos obtidos nas análises financeiras realizadas por Oliveira e Vosti (1997), Sá et al. (2000) e Arco-verde et al. (2003) dos SAFs multiestratos na Amazônia.

T1 Ca-Ba-Pm-CP

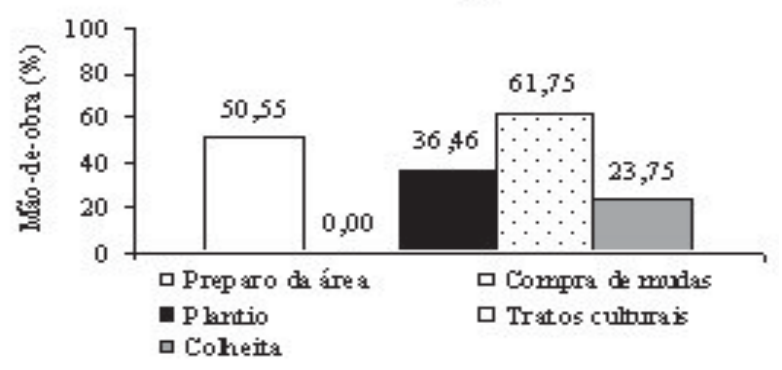

T2 F- $\mathrm{CA}_{1}-\mathrm{B}-\mathrm{Pm}-\mathrm{CP}$

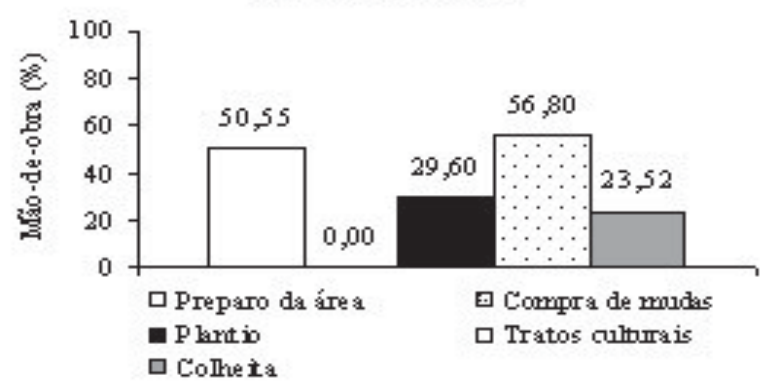

T3 Pu- $\mathrm{Ca}-\mathrm{Ba}-\mathrm{Pm}-\mathrm{Cp}$

Figure 2 - Participation of the total cost (CT) components in the agroforestry systems $\left(T_{1}, T_{2}\right.$ and $\left.T_{3}\right)$ tested in Machadinho d'Oeste, RO-1987 to 2002.

\subsection{Análise financeira}

Utilizando uma taxa anual de desconto de $10 \%$ a.a. no período considerado obteve-se VPL positivo em todos os tratamentos, indicando que os SAFs testados são financeiramente viáveis. Considerando uma situação de mercado em equilíbrio, o SAF $\mathrm{T}_{1}$ foi o tratamento de melhor desempenho financeiro. Levando em conta ainda a ordem de desempenho financeiro, os SAFs $\mathrm{T}_{3}$ e $\mathrm{T}_{2}$ apresentaram-se com as melhores rentabilidades, respectivamente. No SAF $T_{1}$, obteve-se o VPL* de

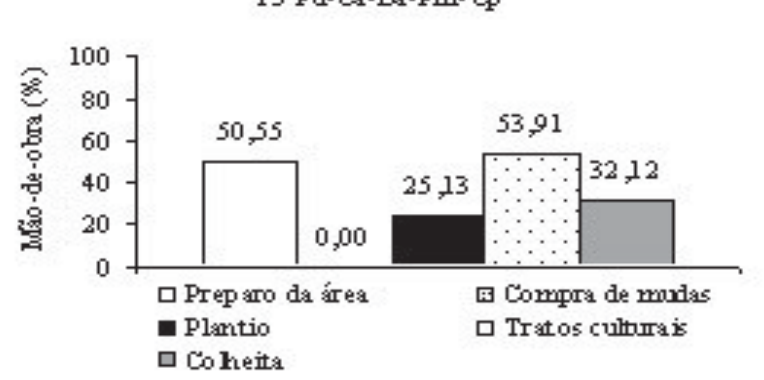

Figura 3 - Participação da mão-de-obra nas diferentes atividades dos sistemas agroflorestais $\left(\mathrm{T}_{1}, \mathrm{~T}_{2}\right.$ e $\left.\mathrm{T}_{3}\right)$ testados em Machadinho d'Oeste, RO -1987 a 2002.

Figure 3 -Labor participation in the differente activities of the agroforestry systems $\left(T_{1}, T_{2}\right.$ and $\left.T_{3}\right)$ tested in Machadinho d'Oeste, RO- 1987 to 2002.

R. Árvore, Viçosa-MG, v.29, n.3, p.401-411, 2005 
Quadro 4 - Análise financeira dos sistemas agroflorestais $\left(\mathrm{T}_{1}, \mathrm{~T}_{2}\right.$ e $\left.\mathrm{T}_{3}\right)$ em Machadinho d'Oeste, RO 1987 a 2002

Table 4 - Financial analysis of the agroforestry systems $\left(T_{1}\right.$, $\mathrm{T}_{2}$ and $\mathrm{T}_{3}$ ) in Machadinho d'Oeste, $\mathrm{RO}-1987$ to 2002

\begin{tabular}{lrrr}
\hline \multicolumn{2}{c}{ Métodos de Avaliação } & \multicolumn{3}{c}{ Sistemas Agroflorestais ${ }^{(1)}$} \\
\cline { 2 - 4 } & \multicolumn{1}{c}{$\mathbf{T}_{\mathbf{1}}$} & \multicolumn{1}{c}{$\mathrm{T}_{2}$} & \multicolumn{1}{c}{$\mathrm{T}_{3}$} \\
\hline VPL $_{\text {(R\$ ha-1 ano-1) }}$ & $\mathbf{3 5 . 8 8 3 , 6 5}$ & $5.334,85$ & $6.584,64$ \\
TIR $_{\text {(\% a.a.) }}$ & $\mathbf{8 6}$ & 19 & 24 \\
VAE $_{\text {(R\$ ha-1 ano-1) }}$ & $\mathbf{4 . 5 8 6 , 5 3}$ & 681,88 & 841,63 \\
VPL $_{\text {(R\$ ha-1 ano-1) }}$ & $\mathbf{4 5 . 8 6 5 , 2 6}$ & $6.818,82$ & $8.416,27$ \\
B/C & $\mathbf{4 , 0 8}$ & 1,44 & 1,51 \\
\hline
\end{tabular}

${ }^{(1)} \mathrm{T}_{1}$ : castanha-do-brasil, banana, pimenta-do-reino e cupuaçu; $\mathrm{T}_{2}$. freijó, banana, pimenta-do-reino e cupuaçu; e $\mathrm{T}_{3}$ : pupunha, banana, pimenta-do-reino e cupuaçu.

$\mathrm{O}$ valor reduzido do lucro no $\mathrm{SAF} \mathrm{T}_{2}$ em relação ao SAF $\mathrm{T}_{1}$ é decorrente da baixa diversificação da produção com esse arranjo de espécies, que apresentou saldo positivo apenas a partir do ano 5 e uma geração de receitas concentrada na comercialização dos frutos de cupuaçu. Os resultados econômicos do SAF $\mathrm{T}_{2}$ foram ligeiramente superiores aos encontrados por Oliveira e Vosti (1997), que, ao analisarem os aspectos econômicos de sistemas agroflorestais com as espécies componentes cupuaçu, freijó e pimenta-do-reino, em Ouro Preto d'Oeste, RO, encontraram VPL de R \$6.540,00 ha-1.
$\mathrm{O} \mathrm{SAF}_{3}$ originou uma receita superior à do $\mathrm{SAF}$ $\mathrm{T}_{2}$, porém o valor reduzido da renda líquida em relação à obtida no $\mathrm{SAF} \mathrm{T}_{1}$ referiu-se à maior oscilação da produção da pupunha quando associada ao cupuaçu. Resultado similar desse sistema foi encontrado por Sá et al. (2000), que analisaram financeiramente sistemas agroflorestais com as espécies castanha-do-brasil, pupunha e cupuaçu, encontrando uma $\mathrm{B} / \mathrm{C}$ de $\mathrm{R} \$ 1,52$.

\subsection{Análise de risco de investimento}

Mediante as simulações feitas pelo @ RISK, o SAF $\mathrm{T}_{1}$ apresentou-se como o SAF de melhor desempenho financeiro, com TIR, VPL, VAE, VPL* e B/C com os valores médios de 85,95\% a.a., $\mathrm{R} \$ 35.958,04 \mathrm{ha}^{-1} \mathrm{ano}^{-1}, \mathrm{R} \$ 4.585,22$ ha $^{-1}$ ano $^{-1}, \mathrm{R} \$ 46.199,53 \mathrm{ha}^{-1} \mathrm{e} 4,08$, respectivamente. Conforme se pode verificar no Quadro 5, a análise de percentis indicou uma probabilidade de $10 \%$ de o VPL* apresentar valores mínimos de R \$39.958,21 ha ano $^{-1}$ e $90 \%$ de probabilidade de exibir valores máximos de $\mathrm{R} \$ 52.972,78$ ha $^{-1}$ ano $^{-1}$, com desvio-padrão de R \$5.026,00. Comparando esses resultados com os dos valores obtidos pelos métodos financeiros utilizados (Quadro 4), pode-se afirmar que o SAF T $\mathrm{T}_{1}$ apresenta elevada viabilidade econômica e menor risco de investimento, considerando que sejam mantidas todas as condições de estabilidade de mercado ao longo do projeto.

Quadro 5 - Estatísticas das variáveis de saída (indicadores financeiros) e de entrada (custos, preço de produtos e taxa de desconto) do sistema agroflorestal $\mathrm{T}_{1}$ : castanha-do-brasil-banana-pimenta-do-reino-cupuaçu em Machadinho d'Oeste, RO - 1987 a 2002

Table 5 - Statistics of output (financial indicators) and input (costs, prices of products and discount rate) variables of the agroforestry system $T_{1}$ : Brasil nut-banana-black pepper-cupuaçu in Machadinho d'Oeste, RO - 1987 to 2002

\begin{tabular}{|c|c|c|c|c|c|c|c|c|c|c|}
\hline \multirow[t]{2}{*}{ Estatísticas } & \multicolumn{4}{|c|}{ Variáveis de Saída } & \multicolumn{6}{|c|}{ Variáveis de Entrada } \\
\hline & $\overline{\text { VPL }}$ & TIR & VAE & VPL* & $\mathrm{B} / \mathrm{C}$ & $\mathrm{C}_{\mathrm{TC}}$ & $\overline{\mathrm{C}_{\mathrm{C}}}$ & $\mathrm{PF}_{\mathrm{Cp}}$ & $\mathrm{PM}_{\mathrm{Ca}}$ & $\overline{\mathrm{TD}}$ \\
\hline Mínimo & $27.540,02$ & 80,95 & $3.795,51$ & $33.158,27$ & 3,33 & 0,80 & 0,80 & 0,80 & 80,12 & 8,01 \\
\hline Máximo & $46.536,04$ & 90,80 & $5.423,31$ & $65.198,09$ & 5,06 & 1,20 & 1,20 & 1,20 & 119,85 & 11,99 \\
\hline Média & $35.958,04$ & 85,95 & $4.585,22$ & $46.199,53$ & 4,08 & 1,00 & 1,00 & 1,00 & 99,98 & 10,00 \\
\hline Desvio-padrão & $2.990,70$ & 1,56 & 288,09 & $5.026,00$ & 0,26 & 0,08 & 0,08 & 0,08 & 8,17 & 0,82 \\
\hline Moda & $32.670,69$ & 82,61 & $4.062,94$ & $40.440,50$ & 3,51 & 0,94 & 0,93 & 0,99 & 90,79 & 8,80 \\
\hline \multicolumn{11}{|c|}{ Percentis } \\
\hline $10 \%$ & $32.166,34$ & 83,89 & $4.197,81$ & $39.958,21$ & 3,75 & 0,89 & 0,89 & 0,89 & 88,90 & 8,90 \\
\hline $20 \%$ & $33.357,20$ & 84,60 & $4.328,29$ & $41.795,34$ & 3,86 & 0,93 & 0,93 & 0,93 & 92,57 & 9,26 \\
\hline $30 \%$ & $34.263,75$ & 85,14 & $4.430,61$ & $43.243,66$ & 3,94 & 0,96 & 0,96 & 0,96 & 95,51 & 9,55 \\
\hline $40 \%$ & $35.076,68$ & 85,56 & $4.511,96$ & $44.569,58$ & 4,01 & 0,98 & 0,98 & 0,98 & 97,87 & 9,78 \\
\hline $50 \%$ & $35.851,89$ & 85,98 & $4.585,36$ & $45.916,07$ & 4,07 & 1,00 & 1,00 & 1,00 & 99,94 & 9,99 \\
\hline $60 \%$ & $36.661,10$ & 86,39 & $4.659,98$ & $47.207,99$ & 4,15 & 1,02 & 1,02 & 1,02 & 102,17 & 10,21 \\
\hline $70 \%$ & $37.532,16$ & 86,81 & $4.746,05$ & $48.652,36$ & 4,22 & 1,05 & 1,04 & 1,04 & 104,52 & 10,45 \\
\hline $80 \%$ & $38.501,50$ & 87,30 & $4.844,18$ & $50.404,37$ & 4,31 & 1,07 & 1,07 & 1,07 & 107,31 & 10,73 \\
\hline $90 \%$ & $39.872,00$ & 87,96 & $4.968,90$ & $52.972,78$ & 4,42 & 1,11 & 1,11 & 1,11 & 110,92 & 11,10 \\
\hline
\end{tabular}

VPL: valor presente líquido, R $\$ \mathrm{ha}^{-1} \mathrm{ano}^{-1}$; TIR: taxa interna de retorno, \%; VAE: valor anual equivalente, R $\$ \mathrm{ha}^{-1}$ ano $^{-1}$; VPL $*$ : valor presente líquido, para o horizonte infinito, $\mathrm{R} \$ . \mathrm{ha}^{-1} \mathrm{ano}^{-1} ; \mathrm{B} / \mathrm{C}$ : benefício/custo; $\mathrm{C}_{\mathrm{TC}}$ : custos dos tratos culturais, \%; CC: custos de colheita, $\% ; \mathrm{PF}_{\mathrm{Cp}}$ : preço do fruto de cupuaçu, \%; $\mathrm{PM}_{\mathrm{Ca}}$ : preço da madeira de castanha-do-brasil, $\mathrm{R} \$ \mathrm{~m}^{-3} ; \mathrm{e}_{\mathrm{TD}}$ : taxa de desconto, $\%$.

R. Árvore, Viçosa-MG, v.29, n.3, p.401-411, 2005 
De acordo com a análise, os valores positivos de elasticidade indicaram uma relação direta entre as variáveis selecionadas $\left(\mathrm{C}_{\mathrm{TC}}\right.$ : custos dos tratos culturais, $\%$; CC: custos de colheita, \%; $\mathrm{PF}_{\mathrm{Cp}}$ : preço do fruto de cupuaçu, \%; $\mathrm{PM}_{\mathrm{Ca}}$ : preço da madeira de castanhado-brasil, $\mathrm{R} \$ \mathrm{~m}^{-3}$; e TD: taxa de desconto, $\%$, ocorrendo efeito inverso quando estas apresentaram valores negativos. Tomando como exemplo as variações do VPL* do SAF $\mathrm{T}_{1}$, pode-se interpretar que, caso ocorra aumento de $10 \%$ na taxa de desconto, haverá diminuição de $8 \%$ sobre o seu valor. No entanto, um aumento de $10 \%$ no preço do fruto de cupuaçu poderá ocasionar incremento de 5\% no VPL* final (Quadro 6), caso a opção seja implantar um SAF com a composição de espécies e a densidade populacional testadas (Quadro 1). A mesma interpretação é válida para os demais métodos testados.

Ainda no Quadro 6 é possível observar que as variáveis que afetaram o VPL*, considerando a ordem de importância $(\mathrm{R})$ individual das variáveis selecionadas, foram: taxa de desconto, preço do fruto de cupuaçu, custo de tratos culturais, preço da madeira de castanhado-brasil e custo de colheita. A mesma interpretação é válida para os demais coeficientes financeiros. Entretanto, a ordem de importância geral $(\mathrm{RG})$ indicou que o preço do cupuaçu foi a variável que mais afetou a análise global. Como complementação da análise, a função densidade de probabilidade simulada para o SAF $\mathrm{T}_{1}$ (Figura 4), que determina com precisão um intervalo de confiança no qual venham a ocorrer os valores desejados ou procurados (PROTIL, 2003), indicou que valores de VPL têm a probabilidade de $15 \%$ de se concentrarem em torno de $\mathrm{R} \$ 35.000 \mathrm{ha}^{-1} . \mathrm{ano}^{-1}$.

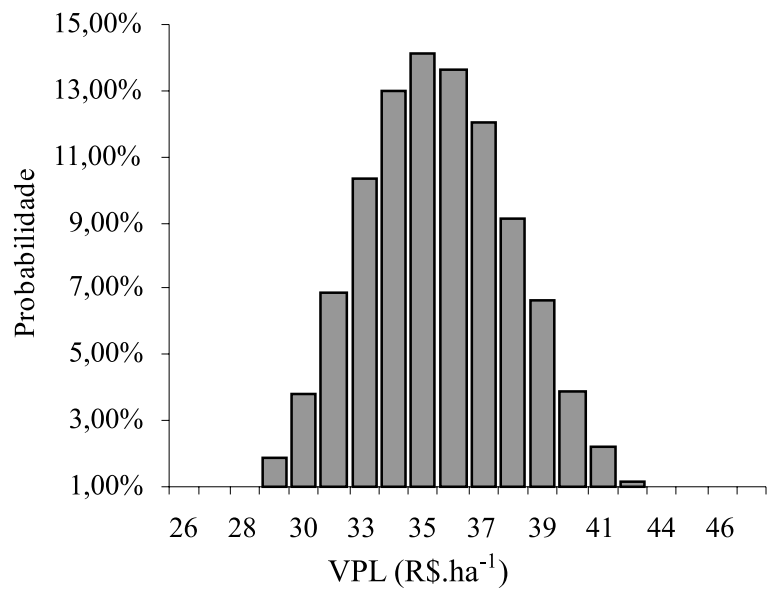

Figura 4 - Probabilidade da distribuição do valor presente líquido - VPL $\left(\mathrm{R} \$\right.$ ha $^{-1} \cdot$ ano $\left.^{-1}\right)$ do sistema agroflorestal $\mathrm{T}_{1}$ : castanha-do-brasil, banana, pimenta-do-reino e cupuaçu em Machadinho d'Oeste, RO - 1987 a 2002.

Figure 4 - Probability distribution of the net present value $-N P V\left(R \$ h^{-1}\right.$ ano $\left.^{-1}\right)$ in agroforestry system $T_{1}$ : Brazil nut-banana-black pepper-сириас̧u in Machadinho d'Oeste, RO - 1987 to 2002.

\section{CONCLUSÕES}

Concluiu-se, portanto, com base nas análises financeiras e de risco de investimento realizadas nos sistemas agroflorestais (SAFs) estudados, que:

- Os SAFs podem ser uma alternativa de investimento para a diversificação da renda e recuperação ambiental para o proprietário rural de Rondônia, com base na composição de espécies e densidades estudadas.

Quadro 6 - Análise de sensibilidade com base nas elasticidades das variáveis de entrada (custos, preço e taxa de desconto), de saída (indicadores financeiros) e ordem de influência na análise do sistema agroflorestal $\mathrm{T}_{1}$ : castanha-dobrasil, banana, pimenta-do-reino e cupuaçu em Machadinho d'Oeste, RO - 1987 a 2002

Table 6 - Sensibility analyses based on the elasticity of the input (costs, price and discount rate), the output (financial indicators) variables, and the influence order on the analysis of the agroforestry system $T_{1}$ : Brasil nut-bananablack pepper-cupuaçu in Machadinho d'Oeste, RO - 1987 to 2002

\begin{tabular}{|c|c|c|c|c|c|c|c|c|c|c|c|}
\hline Variável de entrada & VPL & $\mathrm{R}$ & TIR & $\mathrm{R}$ & VAE & $\mathrm{R}$ & VPL* & $\mathrm{R}$ & $\mathrm{B} / \mathrm{C}$ & $\mathrm{R}$ & RG \\
\hline $\mathrm{C}_{\mathrm{TC}}$ & $-0,136$ & 3 & $-0,577$ & 2 & $-0,181$ & 2 & $-0,104$ & 3 & $-0,550$ & 2 & 2 \\
\hline $\mathrm{C}_{\mathrm{C}}$ & $-0,083$ & 5 & $-0,227$ & 3 & $-0,110$ & 4 & $-0,063$ & 5 & $-0,334$ & 3 & 4 \\
\hline $\mathrm{PF}_{\mathrm{Cp}}$ & 0,728 & 1 & 0,776 & 1 & 0,963 & 1 & 0,557 & 2 & 0,716 & 1 & $\mathbf{1}$ \\
\hline $\mathrm{PM}_{\mathrm{Ca}}$ & 0,095 & 4 & 0,001 & 5 & 0,126 & 3 & 0,073 & 4 & 0,093 & 5 & 5 \\
\hline TD & $-0,653$ & 2 & - & - & $-0,089$ & 5 & $-0,809$ & 1 & $-0,227$ & 4 & 3 \\
\hline
\end{tabular}

$\mathrm{C}_{\mathrm{TC}}$ : custos dos tratos culturais, $\%$; CC: custos de colheita, $\%$; $\mathrm{PF}_{\mathrm{CC}}$ : preço do fruto de cupuaçu, $\%$; $\mathrm{PM}_{\mathrm{Ca}}$ : preço da madeira de castanha-dobrasil, R \$ m ${ }^{-3}$; TD: taxa de desconto, \%; VPL: valor presente líquido, $\mathrm{R} \$$ ha $^{-1}$ ano $^{-1}$; TIR: taxa interna de retorno, \%; VAE: valor anual equivalente, $\mathrm{R} \$$ ha $^{-1} \mathrm{ano}^{-1}$; VPL* : valor presente líquido para o horizonte infinito, $\mathrm{R} \$$ ha $^{-1}$ ano $^{-1}$; B/C: benefício/custo; R: ordem de importância; e RG: ordem de importância (ranking) geral. 
- O SAF T foi o de melhor desempenho financeiro em relação ao SAF $\mathrm{T}_{2}$ e SAF T

freijó, banana, pimenta-do-reino do-reino e cupuaçu, apresentando receitas elevadas desde o primeiro ano de duração do projeto.

- Os custos dos tratos culturais e colheita representaram mais de $70 \%$ da composição dos custos totais, e a participação da mão-de-obra foi superior a 50\% nas fases de preparo da área e de manutenção (tratos culturais) dos SAFs.

- A análise probabilística proporcionada pelo aplicativo @RISK permitiu a determinação, com precisão, de que valores de VPL têm a probabilidade de $15 \%$ de se concentrarem em torno de $\mathrm{R} \$ 35.000 \mathrm{ha}^{-1}$. $\mathrm{ano}^{-1}$, utilizando o arranjo do SAF $\mathrm{T}_{1}$, o que colabora para diminuir as incertezas de investimento nesse tipo de atividade.

- As variáveis que afetaram o VPL*, em ordem de importância (R), foram: taxa de desconto, preço do fruto de cupuaçu, custo de tratos culturais, preço da madeira de castanha-do-brasil e custo de colheita; e o preço do cupuaçu foi a variável que mais afetou a análise financeira global (RG).

- Recomenda-se que os benefícios diretos advindos da utilização do sistema agroflorestal $\mathrm{T}_{1 \text { castanha-do-brasil, }}$ banana, pimenta-do-reino e cupuaçu, em áreas adequadas para sua
implantação no Estado de Rondônia, sejam mais divulgados entre os produtores locais, com atenção à orientação técnica desde o momento de sua implantação.

\section{AGRADECIMENTOS}

À CAPES, por ter possibilitado a participação da autora no Programa de Pós-Graduação do Departamento de Ciências Florestais da Universidade Federal de Viçosa; e a um revisor anônimo, pelos valiosos comentários.

\section{REFERÊNCIAS BIBLIOGRÁFICAS}

ARCO-VERDE, M. F. et al. Avaliação

silvicultural, agronômica e

socioeconômica de sistemas agroflorestais em áreas desmatadas de ecossistemas de mata e cerrado em Roraima. Brasília: PPG-7. 2003, p. 95-99. [on line] Disponível em: <http://www.agrov.com/vegetais/fru/ banana.htm>. Acesso em 20: out. 2003.

R. Árvore, Viçosa-MG, v.29, n.3, p.401-411, 2005
BERGO, C. L.; LUNZ, A. M. P. Cultivo da pupunha para palmito no Acre. Rio Branco: Embrapa Acre, 2000. 15 p. (Embrapa Acre. Circular Técnica, 31).

CALZAVARA, B. B. G. Bananeira. Belém: EMBRAPA-UEPAE Belém, 1989. 12 p. (Embrapa UEPAE Belém. Recomendações Básicas, 8).

CARVAlho, J. E. U. et al. Copoasu (Theobroma grandiflorum (Willd. ex Spreng.) K. Schum.): cultivo y utilizacion. Caracas: Tratado de Cooperación Amazónica, 1999. 151 p.

DUARTE, M. L. R.; POLTRONIERI, M. C. Pimenta-do-reino. [on line] Disponível em: $<$ http://www.cpatu.embrapa.br/pimenta/ pimentadoreino.htm>. Acesso em: 15 out. 2003.

GAMA, M. M. B. Análise técnica e econômica de sistemas agroflorestais em Machadinho d'Oeste, Rondônia. 2003. 112 f. Tese (Doutorado em Ciência Florestal) - Universidade Federal de Viçosa, Viçosa, 2003.

\section{INSTITUTO BRASILEIRO DE GEOGRAFIA E} ESTATÍSTICA - IBGE. Produção agrícola municipal 2001. [on line] Disponível em: <http://www.ibge.gov.br/home/estatistica/ economia/>. Acesso em: 19 nov. 2003.

LOCATELLI, M. Teste de sistemas agroflorestais para o Estado de Rondônia. Porto Velho: EMBRAPA:UEPAE Porto Velho, 1987. 14 p. (Embrapa UEPAE Porto Velho. Projeto de Pesquisa).

LOCATELLI, M. et al. Nutrientes e biomassa em sistemas agroflorestais com ênfase no cupuaçuzeiro, em solo de baixa fertilidade. Porto Velho: Embrapa Rondônia, 2001. 17 p. (Embrapa Rondônia. Boletim de Pesquisa e Desenvolvimento, 1).

MIRANDA, E. E. et al. Diagnóstico agroecológico e sócio-econômico dos produtores rurais de Machadinho d'Oeste-RO em 1999. Campinas: Embrapa Monitoramento por Satélite. 2002. 87 p. (Embrapa Monitoramento por Satélite, Documentos, 18). 
MONTEIRO, R. P. Agricultura familiar: atualização dos coeficientes técnicos para projetos de financiamento em Rondônia. Porto Velho: Embrapa Rondônia, 2002. 39 p. (Embrapa Rondônia. Documentos, 58).

OLIVEIRA, A. D. et al. Análise econômica da exploração, transporte e processamento de madeira de florestas nativas - o caso do município de Jarú, Estado de Rondônia. Cerne, v. 5, n. 2, p. 13-25, 1999.

OLIVEIRA, S. J. M.; VOSTI, S. A. Aspectos econômicos de sistemas agroflorestais em Ouro Preto do Oeste, Rondônia. Porto Velho: Embrapa Rondônia, 1997. 28 p. (Embrapa Rondônia. Circular Técnica, 29).

PALISADE CORPORATION. Risk analysis and simulation add-in for Microsoft Excell or Lotus 1-2-3. New York: Palisade Corporation, 1995. $402 \mathrm{p}$.

Protil, R.M. Aplicação do método Hertz e da Teoria de Oscilação Aleatória de ativos financeiros na modelagem e análise de risco em investimentos florestais. 9p. [on line] Disponível em: <http:// www.anpad.org.br/enanpad2001-trabsapresentados-fin.html>. Acesso em: 22 set. 2003.

RAMÍREZ, G. A. et al. Financial returns, stability and risk of cacao-plantain-timber agroforestry systems in Central America. Agroforestry Systems, n. 51, p. 144-154, 2001.

REZENDE, J. L. P.; OLIVEIRA, A. D. Análise econômica e social de projetos florestais. Viçosa, MG: Universidade Federal de Viçosa, 2001. 389 p.

RODRIGUEZ, L. C. E. Planejamento agropecuário através de um modelo de programação linear não determinista. 1987. 83 f. Dissertação (Mestrado em Economia Agrária) - Escola Superior de Agricultura "Luiz de Queiroz", Piracicaba, 1987.
SÁ, C. P. et al. Análise financeira e institucional dos três principais sistemas agroflorestais adotados pelos produtores do RECA. Rio Branco: Embrapa Acre, 2000. 12 p. (Embrapa Acre. Circular Técnica, 33).

SÁ, C. P. et al. Coeficientes técnicos e custo de implantação da pupunha para palmito no Acre. Rio Branco: Embrapa Acre, 2001. 2 p. (Embrapa Acre. Comunicado Técnico, 33).

SAnTOS, M. J. Avaliação econômica de quatro modelos agroflorestais em áreas degradadas por pastagens na Amazônia Ocidental. 2000. 88 f. Dissertação (Mestrado em Ciências Florestais) Escola Superior de Agricultura "Luiz de Queiroz", Piracicaba, 2000.

SANTOS, M. J.; RODRIGUEZ, L. C. E.; WANDELLI, E. V. Avaliação econômica de quatro modelos agroflorestais em áreas degradadas por pastagens na Amazônia Ocidental. Scientia Forestalis, n. 62, p. 48-61, 2002.

SILVA, I. C. Viabilidade agroeconômica do cultivo do cacaueiro (Theobroma cacao L.) com o açaizeiro (Euterpe oleracea L.) e com a pupunheira (Bactris gasipaes Kunth) em sistema agroflorestal. Floresta, v. 31, n. 1/2, p. 167-168, 2000.

SILVA, M. L.; JACOVINE, L. A. G.; VALVERDE, S. R. Economia florestal. Viçosa, MG: Universidade Federal de Viçosa, 2002. 178 p.

VEnturieri, G. A. Cupuaçu: a espécie, sua cultura, usos e processamento. Belém: Clube do Cupuaçu, 1993. 108 p.

YAMADA, M.; GHOLZ, H. L. Growth and yield of some indigenous trees in an Amazonian agroforestry system: a rural-history-based analysis. Agroforestry Systems, v. 55, p. 17 26, 2002. 